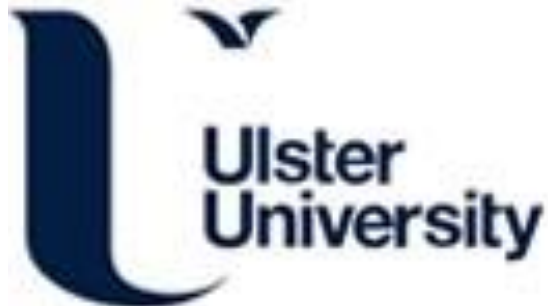

\section{A typology of alcohol consumption among young people - A narrative synthesis}

Davoren, M. P., Cronin, M., Perry, I. J., Demant, J., Shiely, F., \& O'Connor, K. (2016). A typology of alcohol consumption among young people - A narrative synthesis. Addiction Research and Theory, 24(4), $261-273$. https://doi.org/10.3109/16066359.2015.1121244

Link to publication record in Ulster University Research Portal

\section{Published in:}

Addiction Research and Theory

Publication Status:

Published (in print/issue): 01/01/2016

DOI:

10.3109/16066359.2015.1121244

\section{Document Version}

Publisher's PDF, also known as Version of record

\section{General rights}

Copyright for the publications made accessible via Ulster University's Research Portal is retained by the author(s) and / or other copyright owners and it is a condition of accessing these publications that users recognise and abide by the legal requirements associated with these rights.

\section{Take down policy}

The Research Portal is Ulster University's institutional repository that provides access to Ulster's research outputs. Every effort has been made to ensure that content in the Research Portal does not infringe any person's rights, or applicable UK laws. If you discover content in the Research Portal that you believe breaches copyright or violates any law, please contact pure-support@ulster.ac.uk. 


\section{Addiction Research \& Theory}

\section{A typology of alcohol consumption among young people - A narrative synthesis}

\section{Martin P. Davoren, Mary Cronin, Ivan J. Perry, Jakob Demant, Frances Shiely \& Karl O'Connor}

To cite this article: Martin P. Davoren, Mary Cronin, Ivan J. Perry, Jakob Demant, Frances Shiely \& Karl O'Connor (2015): A typology of alcohol consumption among young people - A narrative synthesis, Addiction Research \& Theory, DOI: 10.3109/16066359.2015.1121244

To link to this article: http://dx.doi.org/10.3109/16066359.2015.1121244

曲 Published online: 22 Dec 2015.

Submit your article to this journal $\pi$

Џll Article views: 28

Q View related articles $₫$

View Crossmark data $\nearrow$ 


\title{
A typology of alcohol consumption among young people - A narrative synthesis
}

\author{
Martin P. Davoren ${ }^{a}$, Mary Cronin ${ }^{a}$, Ivan J. Perry ${ }^{a}$, Jakob Demant ${ }^{b}$, Frances Shiely ${ }^{a}$ and Karl O'Connor ${ }^{c}$ \\ ${ }^{\mathrm{a}}$ Department of Epidemiology and Public Health, University College Cork, Cork, Ireland; ${ }^{b}$ Department of Sociology, University of \\ Copenhagen, Copenhagen, Denmark; ' School of Criminology, Politics and Social Policy, University of Ulster, Antrim, Northern Ireland, UK
}

\begin{abstract}
Background Currently, alcohol consumption levels are significantly higher among younger age groups. However, previous research has noted the diversity of motivations and patterns. These patterns of drinking have yet to be synthesised into a typology. The aim of the current study was to synthesise information from studies that produced types of alcohol consumption among young people. Method Quantitative and qualitative literature investigating the different types of drinkers among young people [aged 12-24 years], published in peer reviewed journals, were eligible for inclusion in this systematic review. MEDLINE, PsychInfo and CINAHL were systematically searched for relevant articles published between January 1st 2000 and December 31st 2014. Included papers were critically appraised. A narrative synthesis approach was employed based on guidance from the UK Economic and Social Research Council. Results In total, 13 studies were eligible for inclusion: 11 quantitative, one qualitative and one mixed methods. Six classes of drinkers were formed within this typology. Abstainers reported no alcohol consumption. Light drinkers reported drinking small amounts of alcohol infrequently. In comparison, social and hedonistic drinkers drank most in social situations and to have fun. Heavy and harmful consumers reported increased volume and frequency of consumption including harmful consequences. Conclusion Currently, policy makers are attempting to combat the high levels of harmful alcohol consumption among young people. The current typology provides guidance for targeted interventions in addition to a practical analytic tool in future research.
\end{abstract}

\section{ARTICLE HISTORY}

Received 25 May 2015

Revised 12 November 2015

Accepted 13 November 2015

Published online

9 December 2015

\section{KEYWORDS}

Typology; alcohol;

young people; narrative

synthesis; review

\section{Introduction}

Since the beginning of recorded history alcohol has been consumed for reasons of relaxation, enjoyment and sociability (Rehm et al. 2009). The World Health Organisation reported Europe and America as the heaviest drinking regions in the world (World Health Organization 2014). Previous research has observed that as the mean consumption pattern of the country increases so too does the consumption pattern of the individual (Skog 1985; Rose and Day 1990). Young people (age 18-29) represent a unique sub-section of society who exhibit elevated levels of alcohol consumption (Morgan et al 2009). In 2015, the OECD reported that harmful drinking is on the rise among young people (Sassi 2015). Compounding this, it was recently reported that two-thirds of university students are hazardous alcohol consumers (Davoren et al. 2015) and between one in five (Sassi 2015) and two in five (Murphy et al. 2015) second-level students (age 16-18) report binge drinking. In a recent review of drinking cultures a homogenisation of drinking cultures in Western societies was noted (Gordon et al. 2011). This is supported by similar industry lobbying (Miller and Harkins 2010), technological advances, alcohol advertising (Anderson et al. 2009), alcohol policies (Brand et al 2007) and cultural factors (Gordon et al. 2011) across Western society. Within this culture, research highlights the heterogeneous nature of alcohol consumption as young people exhibit varying consumption patterns. Among the various consumption patterns identified in the literature are young people who abstain from alcohol (O'Connor and Colder 2005). Research also highlights light and moderate levels of alcohol consumption (Hersh and Hussong 2006) in addition to heavy/binge drinkers (Steinhausen and Metzke 2003; Deshpande and RundleThiele 2011) and problem alcohol consumers (Reboussin et al. 2006; Dauber et al. 2009). Public health policy makers have attempted to tackle population consumption throughout the past number of decades using marketing and supply restrictions (Babor and Caetano 2005). However, alcohol remains a major cause of global suffering.

CONTACT Mr. Martin Davoren m.davoren@ucc.ie Department of Epidemiology and Public Health, University College Cork, 4th Floor Western Gateway Building, Western Road, Cork, Ireland

(C) 2015 Taylor \& Francis 
Most recently, typologies have been hypothesised as a pertinent public health tool (Eriksson et al. 2007). The advantage of a typology approach is that it enhances our understanding of a societal phenomenon while making it possible to note patterns across societies. Results from previous research outputs have yet to be synthesised to produce a typology of drinkers. Furthermore, the range of types is not usually considered in policy development and implementation (Berg 2009).

\section{Aim of the study}

The current study aims to conduct a systematic review of previous literature in order to develop a typology of alcohol consumption among young people from research in Western countries.

\section{Methodology}

A narrative synthesis approach was chosen as it allowed for the inclusion of a broad range of research designs (Popay et al. 2006). The narrative synthesis was undertaken using steps outlined in the guidance, developed by Popay et al. (2006) for the UK Economic and Social Research Council (ESRC). This guidance was developed after the Cochrane Collaboration highlighted that 'systematic reviews adopting a narrative approach to synthesis are prone to bias, and may generate unsound conclusions leading to harmful decision' (Popay et al. 2006). Thus, these guidelines aim to 'promote transparent reporting and assessment of the robustness of the results' (Marshall et al. 2012). The guidelines structure the narrative synthesis as follows: (1) identifying a theory, (2) identifying the review process, (3) identifying studies to include in the review, (4) extracting information and completing quality appraisal of included studies and (5) synthesising this information together (Popay et al. 2006).

\section{Identifying a theory}

Skog's theory of the 'Collectivity of Drinking Cultures' (Skog 1985) is utilised as a framework for this narrative synthesis. This theory assumes that an individual's peer group and the culture in which they live impact on an individual's drinking. In addition, it suggests that an individual's alcohol consumption increases as the mean consumption for the society in which they live increases. Skog illustrated that factors influencing an individual's drinking tend to combine multiplicatively and that individual drinking behaviours are regulated by direct and indirect social influences on the individual from their peers and other social and cultural networks
(Skog 1985, 2001). Due to these influences, changes in drinking habits are typically seen as a group phenomenon (Landberg 2010).

\section{Identifying the review process}

Quantitative and qualitative literature, published in peer reviewed journals, investigating the different types of drinkers, among a young adult population (aged 12-24 years) were eligible for inclusion in this review. For quantitative research, articles must have employed factor/cluster analysis to investigate the type of alcohol consumer. These complementary methods are underpinned by an underlying logic of classification, attempting to uncover homogeneous units (Krebs et al. 2000). MEDLINE, PsychInfo and CINAHL were searched for relevant articles separately by quantitative and qualitative research from January 2000 up until December 31st 2014. Full details of the MESH terms and qualitative specific terms (McKibbon et al. 2006) used are outlined in Appendices 1 and 2. No language limits were placed on the inclusion of articles.

\section{Identifying studies to include in the review}

Completed searches were initially title and abstract searched by one reviewer (MPD) and any clearly irrelevant titles were excluded. All papers, which were referred to the research question, were downloaded and fully reviewed. At this point, a final selection was made and any duplicates from the databases were removed. Reference mining was conducted on all included articles. A flow diagram of this is displayed separately for quantitative (Figure 1) and qualitative (Figure 2) findings. English translation of abstracts for relevant papers was available but no full text of a non-English paper was required. References for all included articles were managed in EndNote, a reference package, to keep track of paper selection.

\section{Quality appraisal of included studies}

Included papers were quality assessed using a modified version of the Effective Public Health Practice Project tool for quality assessment of quantitative studies. The appraisal dealt with four main areas: selection bias, study design, data collection methods and analysis. As outlined by Armijo-Olivo et al., sections were rated as strong ( 3 points), moderate ( 2 points) or weak ( 1 point) and an appraisal score out of 12 was defined (Armijo-Olivo et al. 2012). Higher appraisal scores indicated better quality studies. The RATS (Relevance, Appropriateness, Transparency, Soundness) checklist was used for 
Database
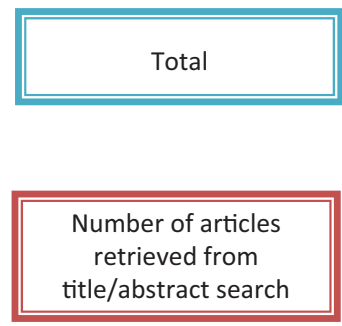

Number of articles retrieved and read in full

[After duplicates across searches were removed]

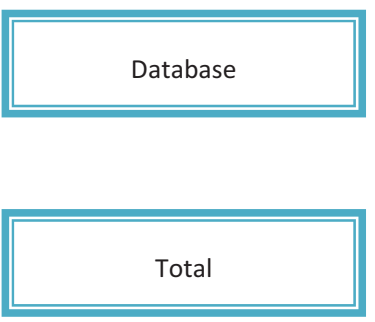

Number of articles retrieved from title/abstract search

Number of articles retrieved and read in full

\section{Total}

[After duplicates across searches were removed]
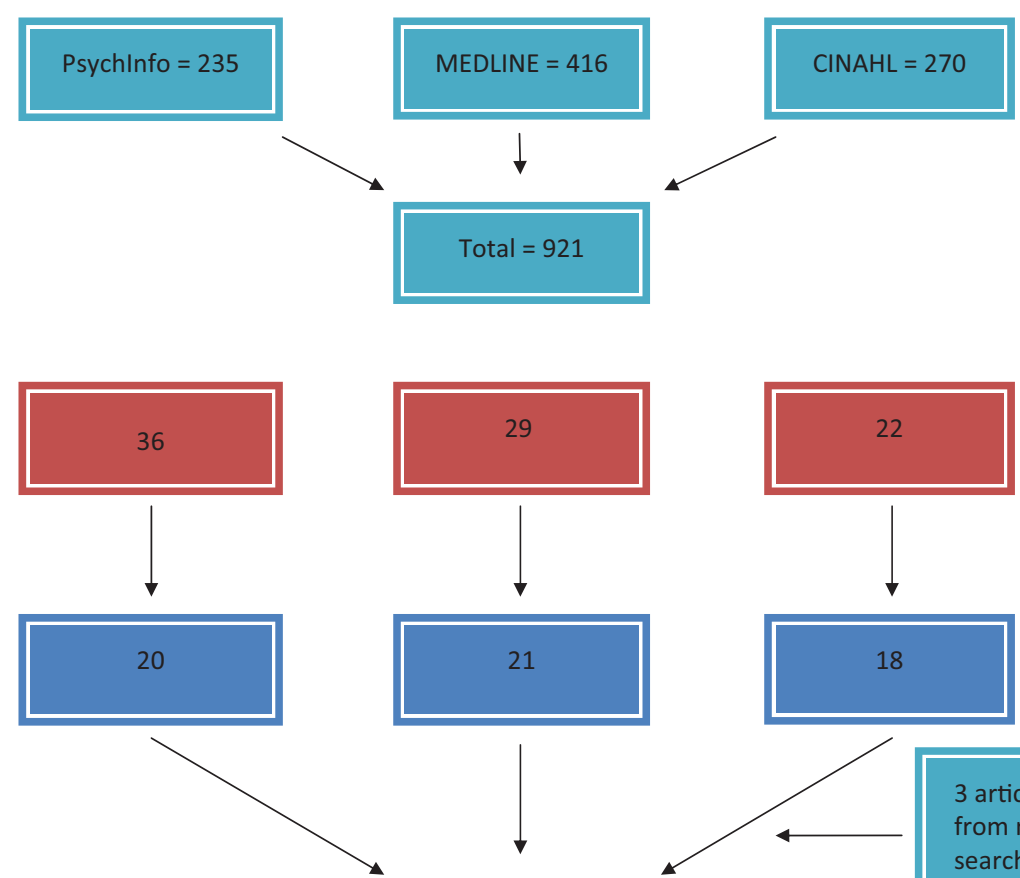

3 articles retrieved from reference searches of included articles

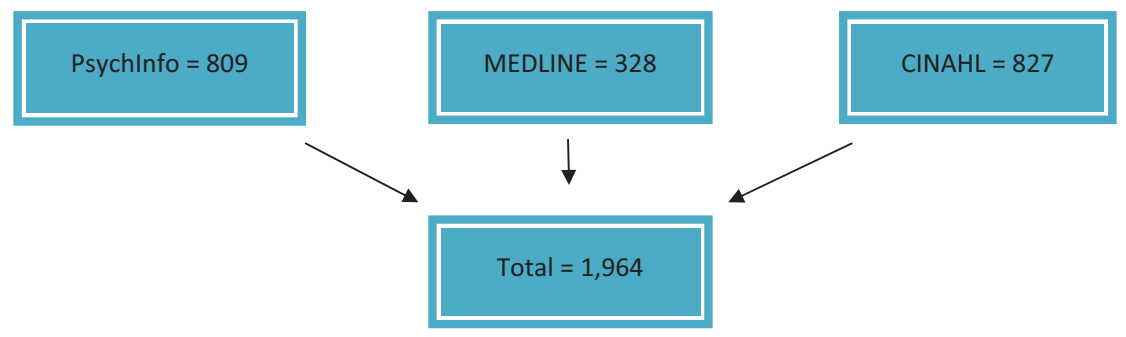

Figure 1. Number of articles retrieved in quantitative research.
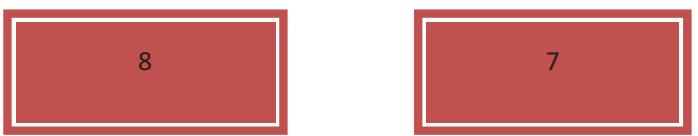

12
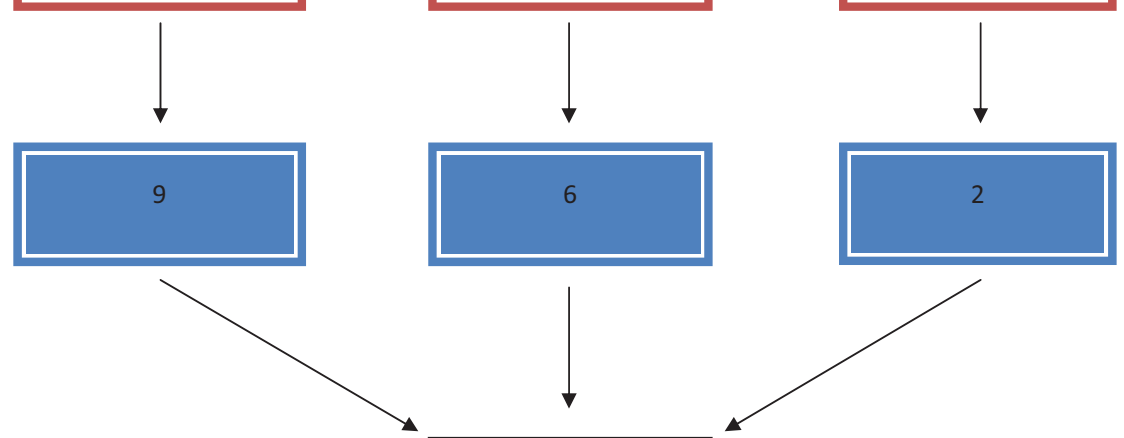

1
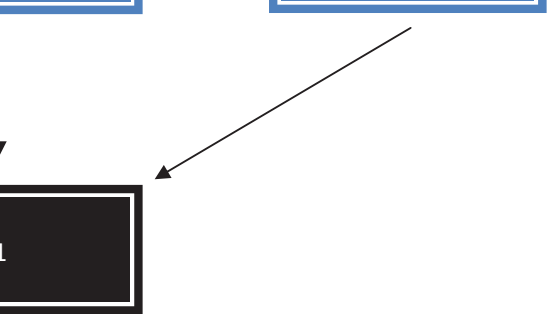

Figure 2. Number of articles retrieved in qualitative research. 
qualitative studies. This checklist comprised of 25 questions about appropriateness of the method and soundness of the approach. As previously described by Leamy et al., each question was dichotomised into yes (1 point) or no (0 points) giving a scale which ranged from poor quality, zero, to high quality, 25 (Leamy et al. 2011). Information regarding the target population, sample size, study methodology and main results were extracted from all included studies (see Table 1). Two authors reviewed articles for quality. Discrepancy in score was discussed with all co-authors to obtain concordance.

\section{Synthesising the information}

Complete results sections from each included article were extracted for analysis. Using NVivo 10, types of drinking behaviour were synthesised from included studies using an inductive approach to content analysis, as it is 'a systematic and objective means of describing and quantifying phenomena' (Elo and Kyngäs 2008). Prevalence of specific types from individual papers was excluded due to the methodological and contextual differences among the studies.

\section{Results}

In total, 13 articles were included in the review; 11 quantitative, one qualitative and one mixed methods study (see Figures 1 and 2). Table 1 details an overview of each included study in this synthesis. The six main types of alcohol consumer identified within this narrative synthesis were Abstainers, Light drinkers, Social drinkers, Hedonistic drinkers, Heavy alcohol consumers and Problem alcohol users. No distinct differences in types reported were observed between countries.

\section{Abstainers}

Young people in this group reported no alcohol consumption either currently (current non-drinkers) or in their lifetime (lifetime abstainers). These individuals reported complete abstention from alcohol. In total, five of the included papers reported a type of drinker who refrained completely from alcohol use (Steinhausen and Metzke 2003; Dauber et al. 2009; Mathijssen et al. 2012; van Lettow et al. 2013; Cleveland et al. 2013). In general, younger people were more likely to be abstainers than older people (Dauber et al. 2009). Three of the included papers excluded non-drinkers as their aim was to investigate drinking styles (Stewart and Power 2002; Reboussin et al. 2006; Comasco et al. 2010).

\section{Light drinkers}

Most studies reported light alcohol users along with young people who have only experimented or sipped alcohol (Stewart and Power 2002; O'Connor and Colder 2005; Reboussin et al. 2006; Percy and Iwaniec 2007; Dauber et al. 2009; Demant \& Törrönen 2011; Craigs et al. 2012; Mathijssen et al. 2012; Cleveland et al. 2013; van Lettow et al. 2013; Jackson et al. 2014). Individuals who reported light levels of alcohol consumption drink small amounts of alcohol and have few alcohol-related problems. These people may be likely to report alcohol consumption but were unlikely to report risky behaviours. Others in this cluster reported sipping alcohol in their lifetime and were unlikely to have three or more drinks on one occasion. This group reported low, infrequent amounts of alcohol consumption. Moreover, individuals in this group were more likely to be younger in age (van Lettow et al. 2013) and female (van Lettow et al. 2013; Jackson et al. 2014) when compared to heavy drinkers. They were described as 'consciously sober' by Mathjssen et al. (2012) and were characterised as cautious, unadventurous and family orientated. Light drinkers reported no instances of heavy drinking.

\section{Social drinkers}

Alcohol facilitates group interactions, meeting new people and feeling a sense of belonging in a group (Comasco et al. 2010; Demant and Törrönen 2011; Jackson et al. 2014). Drinking for social reasons was a clear type of alcohol consumption among these young people (Stewart and Power 2002; Steinhausen and Metzke 2003; Reboussin et al. 2006; Comasco et al. 2010; Demant and Törrönen 2011; Mathijssen et al. 2012). Social drinkers noted drinking at parties with other individuals made them feel more outgoing and social (Comasco et al. 2010). Furthermore, they refer to drinking at bars and other social events in groups with friends (Power et al. 2005) where they drank alcohol due to social expectation.

Social drinking occasions can occur during a social evening away from home, at home or at a friend's house. A sense of mutual solidarity surrounds group drinking, underpinning this type of consumption (Demant and Törrönen 2011). Alcohol consumption facilitates an atmosphere for social activities and feelings of inclusion. Their drinking habits are distinct due to their motivation to consume alcohol being driven by peer influence. This complements Skog's theory which stated that an individual's drinking habits are strongly influenced by the drinking habits of an individual's peers or social network (Skog, 1985, 2001). Social drinkers were more likely to be 


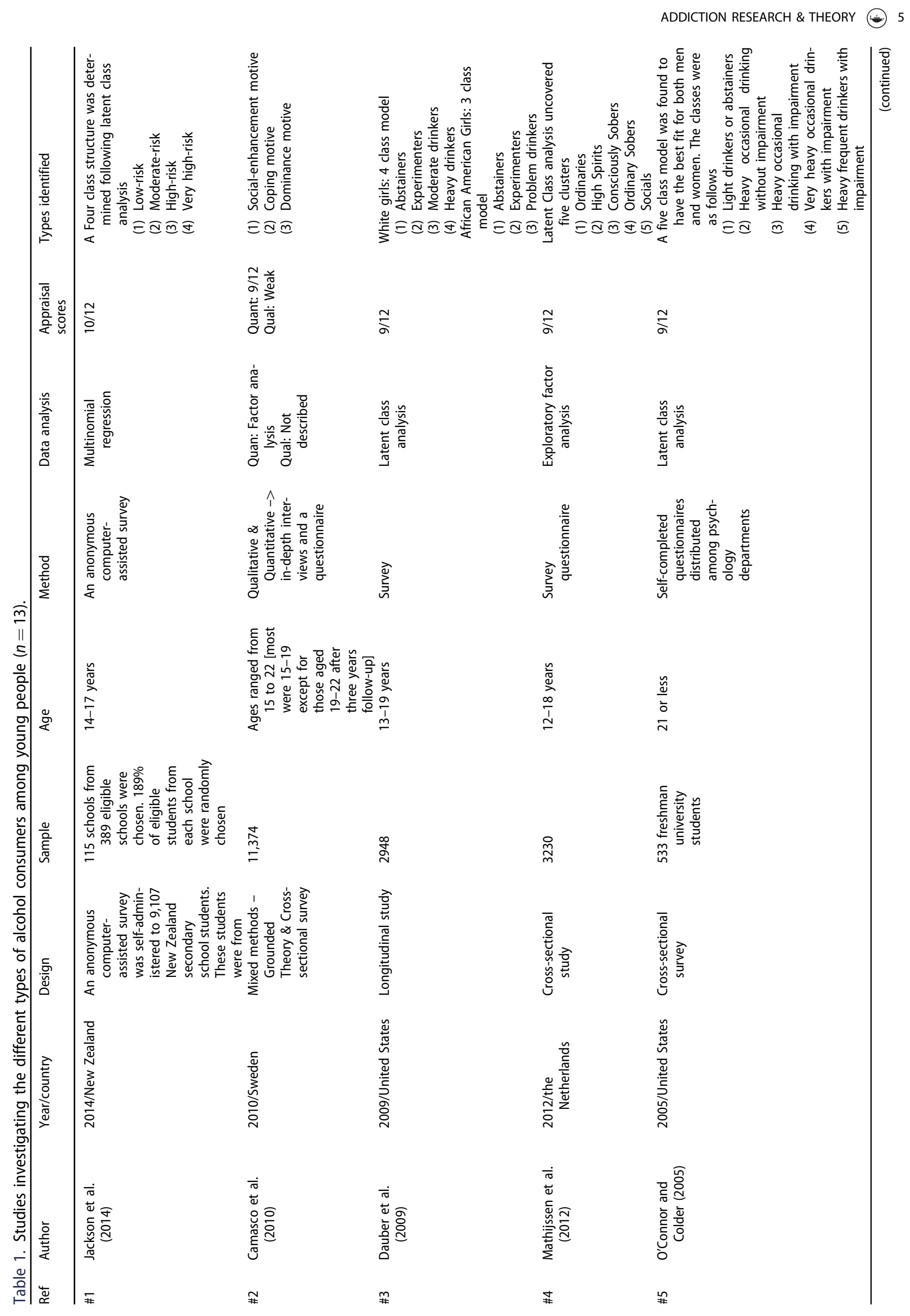


6 M. P. DAVOREN ET AL.

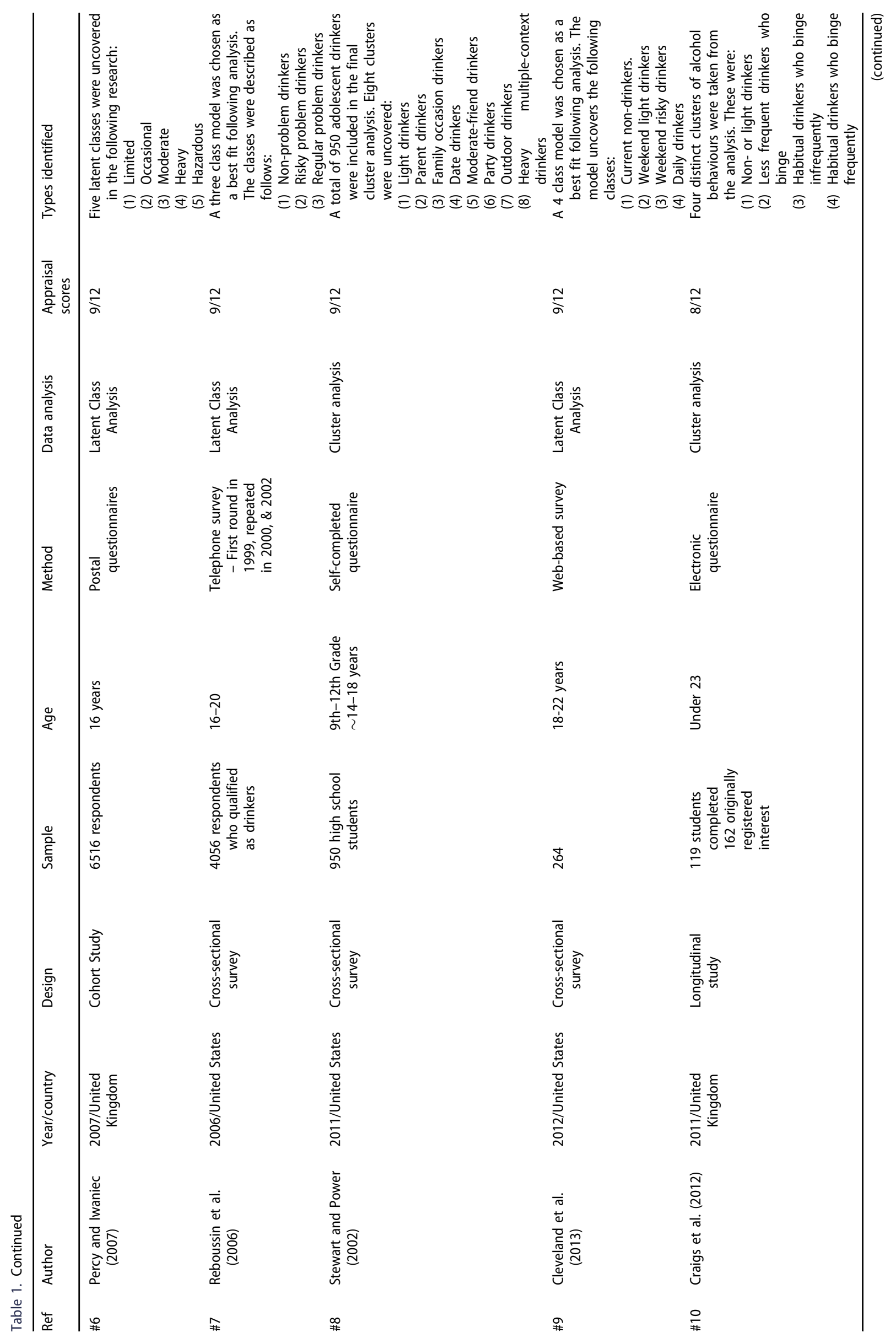


$\underset{\text { in }}{2}$

$\frac{\mathbb{2}}{\frac{\pi}{5}}$
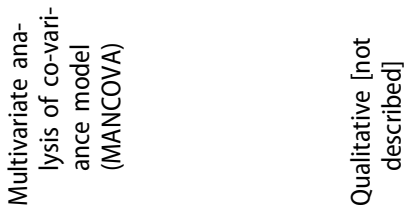

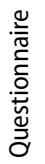

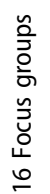

胥

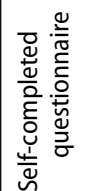

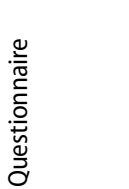

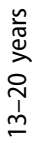

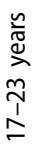

芩

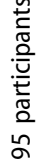

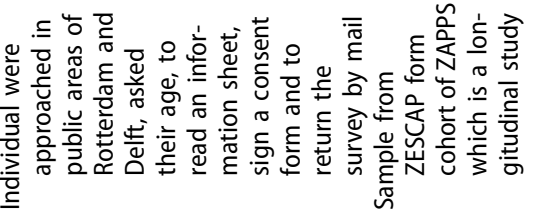
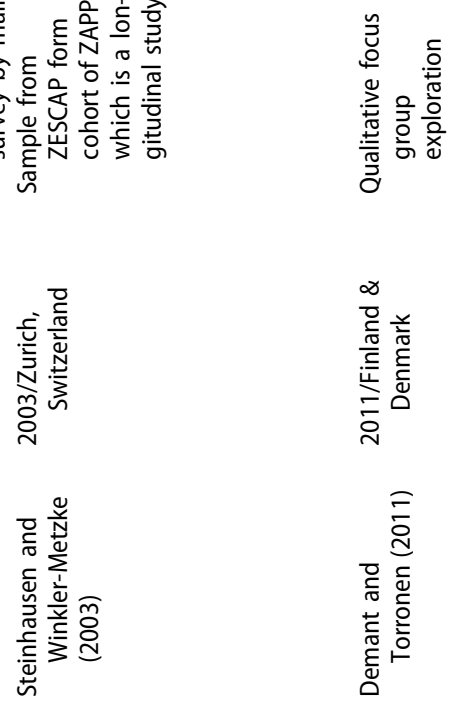

$\cong$

$\stackrel{m}{\#}$ 
female when compared to heavy drinkers (Steinhausen and Metzke 2003) and were older when compared to abstainers (Steinhausen and Metzke 2003; Mathijssen et al. 2012). Individuals in this group report moderate levels of alcohol consumption. This type of drinking was reported in 12 of the included papers.

\section{Hedonistic drinkers}

The majority of the included papers portrayed a hedonistic approach to alcohol use (Stewart and Power 2002; Reboussin et al. 2006; Dauber et al. 2009; Comasco et al. 2010; Demant and Törrönen 2011; Craigs et al. 2012; Mathijssen et al. 2012; Cleveland et al. 2013; van Lettow et al. 2013). Hedonism is the view that pleasure is the only good thing in life and can be defined by viewing 'pleasure as the only possible object of desire, because all motivation is based on the prospect of pleasure' (O'Shaughnessy and O'Shaughnessy 2002). An integral part of hedonistic drinking is the use of alcohol to change one's mood, for enjoyment, to enhance social situations, to reduce inhibitions and to get intoxicated and experiment (Comasco et al. 2010). These individuals enjoyed being drunk and drank to feel pleasure (van Lettow et al. 2013). This style of drinking is associated with impulsive drinking behaviours and drinking larger amounts of alcohol with the intention of having fun and enjoyment (Stewart and Power 2002). This group of drinkers are distinct from social drinkers in their selfindulgent motivation to consume alcohol. Instead of being motivated by peers and social groups, hedonistic drinkers are driven by their personal need to feel pleasure, overcome reservations and enjoy themselves. These individuals are more likely to be older than light drinkers (Craigs et al. 2012), and less likely to be female than other groups (van Lettow et al. 2013).

\section{Heavy drinkers}

The majority of included papers (12) discussed patterns of heavy alcohol consumption among young people. Heavy alcohol consumption ranged in name from 'weekend risky drinker' to 'habitual drinkers'; describing individuals who would consume harmful levels alcohol on a regular basis. Individuals who fell within heavy alcohol consumption groups would report heavy drinking, high rates of consequences due to alcohol consumption, high risk drinking behaviours and regular patterns of alcohol consumption (Stewart and Power 2002; Steinhausen and Metzke 2003; O'Connor and Colder 2005; Reboussin et al. 2006; Percy and Iwaniec 2007; Dauber et al. 2009; Comasco et al. 2010; Demant and Törrönen 2011; Craigs et al. 2012; Mathijssen et al. 2012; Cleveland et al.
2013; Jackson et al. 2014). This group would consume higher amounts of alcohol and consume alcohol more frequently than their peers who report light drinking or social levels of drinking. Heavy drinkers were associated with early initiation into alcohol consumption, drinking to intoxication and binge drinking (Stewart and Power 2002; Reboussin et al. 2006; Demant and Törrönen 2011; Cleveland et al. 2013). Adverse consequences for this type of alcohol consumer are reported in the literature, including hangovers, blackouts, getting sick, feeling depressed, losing control and having an outburst of anger. In general, men (Reboussin et al. 2006; Sacco et al. 2009) and those at the upper age limit of this study (Steinhausen and Metzke 2003) were more likely to report heavy drinking.

\section{Problem alcohol users}

Problem alcohol users and the negative aspects associated with this type of alcohol use were described in 11 of the 13 included studies (Stewart and Power 2002; Reboussin et al. 2006; Percy and Iwaniec 2007; Dauber et al. 2009; Comasco et al. 2010; Demant and Törrönen 2011; Craigs et al. 2012; Mathijssen et al. 2012; Cleveland et al. 2013; van Lettow et al. 2013; Jackson et al. 2014). Problem alcohol users are described as individuals who reported solitary drinking, drinking in the morning, individuals who drink on a daily basis and those who report consuming alcohol and driving (Stewart and Power 2002; Dauber et al. 2009; Cleveland et al. 2013). A number of papers also reported their drinking pattern placing them in unwanted sexual situations or regretting sex (Dauber et al. 2009; Cleveland et al. 2013). Problem alcohol users are described as such because of the negative outcomes associated with their alcohol consumption.

In addition, a number of studies highlighted that problem drinkers are fuelled by negative emotions and a wish to change or improve mood through heavy alcohol consumption. Physiological and pharmacological sideeffects among problem alcohol users are frequent. They describe drinking to think of something else/to forget my worries, my problems' (Comasco et al. 2010). Compared to abstainers/light drinkers this group is older (Jackson et al. 2014) and predominantly male (Percy and Iwaniec 2007; Jackson et al. 2014).

\section{Discussion}

Alcohol consumption is a leading cause of suffering in society (World Health Organization 2009). Reports from different countries reveal that young people today drink more, with increasing emphasis on binge drinking and drunkenness than earlier generations (McCabe 2002; 
Carey et al. 2007; Murphy and Murphy 2010). This is said to have increased over the last 45 years. Authors have previously hypothesised this as the 'psychoactive revolution' and by the 1990s, a decade defined by a 'new culture of intoxification' had manifested, peaking in 2001 (Järvinen and Room 2007). This cultural shift is further compounded by the fact that young adult consumption levels remained steady for the 1970s and 80s but doubled in the 1990s (Järvinen and Room 2007). The current research describes a synthesis of previously published typologies of alcohol consumption identified in young people living in Western society. Systematic reviews furnish policy makers with the entire range of relevant findings from research on a particular topic, ensuring they are not misguided by the results of one or two studies (Akobeng 2005). The authors believe this review will be a robust analytic tool in future research in addition to providing novel information for public policy makers when tailoring health promotion interventions.

Abstainers and light drinkers may serve as protective and moderating factors when socialising among peers. Recently, an Australian report mooted that these individuals could be described as controlled and conscientious (VicHealth 2013). These individuals look out for others when socialising. Health promotion practitioners have recommended that these individual's drinking attitudes should be 'encouraged and supported as the ideal drinking attitude' (VicHealth 2013). The protection peers offer each other against engagement in excessive alcohol consumption should be emphasised (Quinn and Bussey 2015). This can be achieved by 'challenging society's negative image of moderate drinking and empowering people to abstain or drink less' (VicHealth 2013).

As previously noted, 'the classes of drinking occasions when heavy drinking occurs are of special interest from the preventive perspective' (Mustonen et al. 2014). Skog emphasises, 'egos drinking is very strongly influenced by alter's drinking in group sessions' (Skog 1985) highlighting the importance of an individual's peer group and culture on their drinking pattern (Skog 1985). This complements the current research where the influence of peer drinking is evident. Young people highlight their motivators in terms of 'house parties', excessive drinking and enjoyment which is apparent in social, hedonistic and heavy consumers. These correspond to the previously described motives: 'social' and 'enhancement' (Stewart et al. 1996; Quinn and Bussey 2015).

Individuals anticipate more arousal from risky behaviours (Katz et al. 2000), complementing our finding of hedonistic drinking. Hedonistic drinking has previously been noted as a culture of alcohol consumption among our society (Hurlbut and Sher 1992; Gordon et al. 2011). It is sustained by a technological era of instant gratification to which young people have been excessively exposed. As outlined by Gordon, 'rules governing drinking behaviours vary and are often informal, socially negotiated and acquired via the socialisation process' (Gordon et al. 2011). It is these social cultures, which govern and reflect attitudes toward alcohol. For example, Germany tends to value individuals who can hold one's drink while the UK appears to celebrate intoxication and high levels of alcohol consumption. Gordon defines these variations in "levels of drinking and drunkenness as the "hedonism" dimension to drinking cultures' (Gordon et al. 2011). These cultural norms represent an important way of regulating drinking behaviour.

\section{Recommendations for policy \& health promotion strategies}

Public policy makers have attempted to combat alcohol use with a number of legislative measures. Despite this, consumption levels have continued to increase (Davoren et al. 2015). This typology gives us further insight into the drinking patterns of young people. For example, abstainers give insight into their reasons for not consuming alcohol (Steinhausen and Metzke 2003; Dauber et al. 2009; Mathijssen et al. 2012), while light drinkers give insight into a form of conscious experimentation which occurs as young people explore alcohol consumption while remaining conscious and in control of their bodies (Hersh and Hussong 2006; Reboussin et al., 2006; Dauber et al. 2009; Huang et al. 2011; Mathijssen et al. 2012). These consumption patterns are also observed in comparatively older populations (Sacco et al. 2009), and in other risk taking activities (Fountain et al. 1999). Although, harms caused by these drinking styles are negligible, national policy and health promotion strategies aimed toward education, taxation and restrictions on opening hours remain crucial to ensuring these individuals do not transition to heavier patterns of alcohol consumption. In addition, the socio-cultural norms and practices upheld by these groups are useful in the tailoring positive health promotion strategies, aimed at altering the negative image of abstinence and light drinking among young people (Quinn and Bussey 2015).

In the past decade a surge in predrinking has been observed with adolescents predrinking before parties or before going out (Kuntsche and Gmel 2013). This was observed in a typology which was formed almost 50 years ago (Park 1967). This is being fuelled by the availability of cheap alcohol through off-sales and the current phenomenon of the 'house party' (Wells et al. 2009). The most effective measure to reduce the harms caused by this style of drinking is minimum unit pricing. This measure would increase the price of off-licence sales 
thus reducing the consumption among hedonistic drinkers (The Scottish Government 2013). A reduction in the density of outlets surrounding colleges, universities and schools would also reduce consumption and patterns of harm. Culturally, a ban on the alcohol industry providing sports sponsorship would break the view of alcohol as a cultural artefact (McGee 2013). However, for young people reporting problem drinking and addictive tendencies, their motives for drinking are centred on coping (Comasco et al. 2010). Individual strategies will be required among these individuals, such as brief intervention therapy, support structures and clear pathways of referral to addiction clinics.

Many screening tools employed by health professionals define young people as either hazardous or nonhazardous drinkers. The protection of this vulnerable population would benefit from the development of a screening tool which incorporated the typology presented in this synthesis. This typology would aid the health professional to tailor brief interventions and advice, thus improving patient care (Winograd et al. 2012). In addition, health promotion practitioners should employ the current review to understand the culture of alcohol consumption further by tailoring effective health promotion strategies to influence these specific groups and reduce consumption (Santos 2013).

\section{Strengths and limitations}

This synthesis has a number of strengths. A range of databases were reviewed to obtain the breath of literature available researching drinking types. Relevant articles, regardless of language were considered. No previous typology had been developed. As studies assessing drinking type had utilised quantitative and qualitative research, a robust process was used to reduce the weaknesses of a narrative syntheses approach (Popay et al. 2006).

However, the synthesis is limited to the published data which tends to range in quality. Most of the included studies were cross-sectional. Moreover, the synthesis was constrained by the broad age limits used in previous research. Those aged less than 18 will report a distinctly different alcohol consumption compared to their peers aged 18 years and older. Furthermore, policy implications for those above and below the legal drinking age will be distinctly different. Research questions in the included articles were distinct across each study. The impact of this is difficult to control. Finally, answers may be influenced by recall and/or social-desirability bias due to participants disclosing a range of risk-taking behaviour.

A typology itself can be described as a 'systematic method for classifying similar events, actions, objects, people or places into distinct groupings' (Berg 2009). The main objective of a typology is to provide additional information on the material to the reader. This is executed by attempting mutually exclusive categories, making sure all elements have been accounted for and ensuring the categories make theoretically meaningful appraisals of the literature. However, the idea of a typology can be a reductionist one, loosing nuances from data which may better describe the different aspects of alcohol consumption. Many argue this is the beauty of the method as it 'permits the researcher to present the data in an organised and simple fashion, allowing the reader to better understand the explanations offered' (Berg 2009).

\section{Conclusion}

The current synthesis outlines a typology of alcohol consumption among young people in Western societies. It displays a varied prevalence of reported alcohol use for specific groups of young people implying that the need for alcohol interventions is not uniform. Thus, public policy should address the specific needs of each of the targeted groups (Francis et al. 2014) through a mix of population-level and individual measures. Future public policy should consider this typology when developing alcohol interventions, screening tools and tailoring motivational interventions.

\section{Declaration of interest}

The authors declare that there are no competing interests.

\section{References}

Akobeng A. 2005. Understanding systematic reviews and meta-analysis. Arch Disease Child. 90(8):845-848.

Anderson P, De Bruijn A, Angus K, Gordon R, Hastings G. 2009. Impact of alcohol advertising and media exposure on adolescent alcohol use: a systematic review of longitudinal studies. Alcohol Alcohol 44(3):229-243.

Armijo-Olivo S, Stiles CR, Hagen NA, Biondo PD, Cummings GG. 2012. Assessment of study quality for systematic reviews: a comparison of the Cochrane Collaboration Risk of Bias Tool and the Effective Public Health Practice Project Quality Assessment Tool: methodological research. J Eval Clin Pract. 18(1):12-18.

Babor TF, Caetano R. 2005. Evidence-based alcohol policy in the Americas: strengths, weaknesses, and future challenges. Rev Panam Salud Pública 18(4-5):327-337.

Berg BL. 2009. Qualitative research methods for the social sciences. Vol. 7. MA: Allyn \& Bacon.

Brand DA, Saisana M, Rynn LA, Pennoni F, Lowenfels AB. 2007. Comparative analysis of alcohol control policies in 30 countries. PLoS Med. 4(4):e151. 
Carey KB, Scott-Sheldon LAJ, Carey MP, DeMartini KS. 2007. Individual-level interventions to reduce college student drinking: a meta-analytic review. Addict Behav. 32(11):2469-2494.

Cleveland MJ, Mallett KA, White HR, Turrisi R, Favero S. 2013. Patterns of alcohol use and related consequences in non-college-attending emerging adults. J Stud Alcohol Drugs 74(1):84-93.

Comasco E, Berglund K, Oreland L, Nilsson KW. 2010. Why do adolescents drink? Motivational patterns related to alcohol consumption and alcohol-related problems. Subst Use Misuse 45(10):1589-1604.

Craigs CL, Bewick BM, Gill J, O’May F, Radley D. 2012. UK student alcohol consumption: A cluster analysis of drinking behaviour typologies. Health Educ J. 71(4):516-526.

Dauber S, Hogue A, Paulson JF, Leiferman JA. 2009. Typologies of alcohol use in White and African American adolescent girls. Subst Use Misuse 44(8):1121-1141.

Davoren MP, Shiely F, Byrne M, Perry IJ. 2015. Hazardous alcohol consumption among university students in Ireland: a cross-sectional study. BMJ Open. 5(1):e006045.

Demant J, Törrönen J. 2011. Changing drinking styles in Denmark and Finland. Fragmentation of male and female drinking among young adults. Subst Use Misuse 46(10):1244-1255.

Deshpande S, Rundle-Thiele S. 2011. Segmenting and targeting American university students to promote responsible alcohol use: a case for applying social marketing principles. Health Market Quart. 28(4):287-303.

Elo S, Kyngäs H. 2008. The qualitative content analysis process. J Adv Nurs. 62(1):107-115.

Eriksson Å, Tengström A, Hodgins S. 2007. Typologies of alcohol use disorders among men with schizophrenic disorders. Addict Behav. 32(6):1146-1163.

Fountain J, Bartlett H, Griffiths P, Gossop M, Boys A, Strang J. 1999. Why say no? Reasons given by young people for not using drugs. Addict Res Theory 7(4):339-353.

Francis JM, Grosskurth H, Changalucha J, Kapiga SH, Weiss HA. 2014. Systematic review and meta-analysis: prevalence of alcohol use among young people in eastern Africa. Trop Med Int Health 19(4):476-488.

Gordon R, Heim D, MacAskill S. 2011. Rethinking drinking cultures: a review of drinking cultures and a reconstructed dimensional approach. Public Health Addict Res Theory 126(1):3-11.

Hersh MA, Hussong AM. 2006. High school drinker typologies predict alcohol involvement and psychosocial adjustment during acclimation to college. J Youth Adolesc. 35(5):738-751.

Huang J-H, DeJong W, Schneider SK, Towvim LG. 2011. Endorsed reasons for not drinking alcohol: a comparison of college student drinkers and abstainers. J Behav Med. 34(1):64-73.

Hurlbut SC, Sher KJ. 1992. Assessing alcohol problems in college students. J Am Coll Health 41(2):49-58.

Jackson N, Denny S, Sheridan J, Fleming T, Clark T, Teevale T, Ameratunga S. 2014. Predictors of drinking patterns in adolescence: a latent class analysis. Drug Alcohol Depend. 135:133-139.

Järvinen M, Room R. 2007. Youth drinking cultures: European experiences. Vol. 13. United Kingdom: Ashgate Publishing, Ltd.
Katz EC, Fromme K, D’Amico EJ. 2000. Effects of outcome expectancies and personality on young adults' illicit drug use, heavy drinking, and risky sexual behavior. Cog Therapy Res. 24(1):1-22.

Krebs D, Berger M, Ferligoj A. 2000. Approaching achievement motivation-comparing factor analysis and cluster analysis. New approaches in applied statistics. Metodoloski zvezki. 16:147-171.

Kuntsche E, Gmel G. 2013. Alcohol consumption in late adolescence and early adulthood - where is the problem. Swiss Med Wkly. 143:w13826.

Landberg J. 2010. Alcohol-related problems in Eastern Europe: a comparative perspective. Stockholm. University, Centre for Social Research on Alcohol and Drugs.

Leamy M, Bird V, Le Boutillier C, Williams J, Slade M. 2011. Conceptual framework for personal recovery in mental health: systematic review and narrative synthesis. $\mathrm{Br} \mathrm{J}$ Psychiatry 199(6):445-452.

Marshall IJ, Wolfe CD, McKevitt C. 2012. Lay perspectives on hypertension and drug adherence: systematic review of qualitative research. Br Med J. 345 doi: http://dx.doi.org/ 10.1136/bmj.e3953.

Mathijssen J, Janssen M, van Bon-Martens M, van de Goor I. 2012. Adolescents and alcohol: an explorative audience segmentation analysis. BMC Public Health 12(1):742.

McCabe SE. 2002. Gender differences in collegiate risk factors for heavy episodic drinking. J Stud Alcohol Drugs 63(1):49.

McGee H. 2013. Ministers favouring alcohol sports ban told to find new funds; [cited 2013 Dec 20]. Available from: http:// www.irishtimes.com/news/politics/ministers-favouring-alcohol-sports-ban-told-to-find-new-funds-1.1477873.

McKibbon KA, Wilczynski NL, Haynes RB. 2006. Developing optimal search strategies for retrieving qualitative studies in PsycINFO. Eval Health Prof. 29(4):440-454.

Miller D, Harkins C. 2010. Corporate strategy, corporate capture: food and alcohol industry lobbying and public health. Crit Social Policy 30(4):564-589.

Morgan K, McGee H, Dicker P, Brugha R, Ward M, Shelley E, Van Lente E, Harrington J, Barry M, Perry I, et al. 2009. SLÁN 2007: survey of lifestyle, attitudes and nutrition in Ireland. Alcohol use in Ireland: a profile of drinking patterns and alcohol-related harm from SLÁN 2007. Dublin: Department of Health and Children.

Murphy F, Murphy M. 2010. The impact of social marketing on Irish female college binge drinking: Are fear appeals affective. Paper presented at the World Social Marketing Conference, United Kingdom.

Murphy E, O’Sullivan I, O’Donovan D, Hope A, Perry IJ, Davoren MP. 2015. Alcohol consumption: does the apple fall far from the tree? Cork, Ireland: Department of Epidemiology \& Public Health, University College Cork.

Mustonen H, Mäkelä P, Lintonen T. 2014. Toward a typology of drinking occasions: latent classes of an autumn week's drinking occasions. Addict Res Theory 22(6):524-534.

O'Connor RM, Colder CR. 2005. Predicting alcohol patterns in first-year college students through motivational systems and reasons for drinking. Psychol Addict Behav. 19(1):10-20.

O’Shaughnessy J, O’Shaughnessy NJ. 2002. Marketing, the consumer society and hedonism. Eur J Market. 36(5/6):524-547.

Park P. 1967. Dimensions of drinking among male college students. Soc Probs. 14(4):473-482. 
Percy A, Iwaniec D. 2007. The validity of a latent class typology of adolescent drinking patterns. Irish J Psychol Med. 24(1):13-18.

Popay J, Roberts H, Sowden A, Petticrew M, Arai L, Rodgers M, Duffy S. 2006. Guidance on the conduct of narrative synthesis in systematic reviews. A product from the ESRC methods programme. Version, 1.

Power TG, Stewart CD, Hughes SO, Arbona C. 2005. Predicting patterns of adolescent alcohol use: a longitudinal study. J Stud Alcohol Drugs 66(1):74-81.

Quinn CA, Bussey K. 2015. Adolescents' anticipated social outcomes for drinking alcohol and being drunk. Addict Res Theory. 23(3):253-264.

Reboussin BA, Song E-Y, Shrestha A, Lohman KK, Wolfson M. 2006. A latent class analysis of underage problem drinking: evidence from a community sample of 16-20 year olds. Drug Alcohol Depend. 83(3):199209.

Rehm J, Mathers C, Popova S, Thavorncharoensap M, Teerawattananon Y, Patra J. 2009. Global burden of disease and injury and economic cost attributable to alcohol use and alcohol-use disorders. Lancet 373(9682):2223-2233.

Rose G, Day S. 1990. The population mean predicts the number of deviant individuals. $\mathrm{Br}$ Med J. 301(6759):1031.

Sacco P, Bucholz KK, Spitznagel EL. 2009. Alcohol use among older adults in the national epidemiologic survey on alcohol and related conditions: a latent class analysis. J Stud Alcohol Drugs. 70(6):829-838.

Santos CR. 2013. Consumption culture in Europe: insight into the beverage industry. Hershey, PA: IGI Global.

Sassi F. 2015. Tackling harmful alcohol use: economics and public health policy. France: OECD Publishing.

Skog OJ. 1985. The collectivity of drinking cultures: a theory of the distribution of alcohol consumption. $\mathrm{Br} \mathrm{J}$ Addict. 80(1):83-99.
Skog O-J. 2001. Commentary on Gmel \& Rehm's interpretation of the theory of collectivity of drinking culture. Drug Alcohol Rev. 20(3):325-331.

Steinhausen HC, Metzke CW. 2003. The validity of adolescent types of alcohol use. J Child Psychol Psychiatry. 44(5): 677-686.

Stewart C, Power TG. 2002. Identifying patterns of adolescent drinking: a tri-ethnic study. J Stud Alcohol Drugs 63(2):156.

Stewart SH, Zeitlin SB, Samoluk SB. 1996. Examination of a three-dimensional drinking motives questionnaire in a young adult university student sample. Behav Res Therapy 34(1):61-71.

The Scottish Government. 2013. Minimum pricing; [cited 2013 Oct 20]. Available from: http://www.scotland.gov.uk/Topics/ Health/Services/Alcohol/minimum-pricing

van Lettow B, Vermunt JK, Vries H, Burdorf A, Empelen P. 2013. Clustering of drinker prototype characteristics: What characterizes the typical drinker? Br J Psychol. 104(3):382-399.

VicHealth. 2013. Drinking-related lifestyles: exploring the role of alcohol in Victorians' lives In: Victorian Health Promotion Foundation, editor. Melbourne, Australia: RMIT University.

Wells S, Graham K, Purcell J. 2009. Policy implications of the widespread practice of 'pre-drinking' or 'pre-gaming' before going to public drinking establishments-are current prevention strategies backfiring? Addiction 104(1):4-9.

Winograd RP, Littlefield AK, Martinez J, Sher KJ. 2012. The drunken self: the five-factor model as an organizational framework for characterizing perceptions of one's own drunkenness. Alcohol Clin Exp Res 36(10):1787-1793.

World Health Organization. 2009. Global health risks: mortality and burden of disease attributable to selected major risks. Geneva, Switzerland: World Health Organization.

World Health Organization. 2014. Global status report on alcohol and health-2014. Geneva, Switzerland: World Health Organization.

Appendix 1. Search terms from Psychlnfo [Quantitative].

\begin{tabular}{|c|c|c|}
\hline & Search term & $\begin{array}{l}\text { Number of } \\
\text { articles retrieved }\end{array}$ \\
\hline S14 & S12 AND S13 & 190 \\
\hline S13 & $\begin{array}{l}\text { MM 'Alcohol Drinking Attitudes' OR MM 'Alcohol Drinking Patterns' OR MM 'Alcohol Abuse' OR MM 'Alcohol } \\
\text { Intoxication' OR MM 'Social Drinking' }\end{array}$ & 26,196 \\
\hline S12 & S6 AND S11 & 14,077 \\
\hline S11 & S7 OR S8 OR S9 OR S10 & 669,066 \\
\hline S10 & $\begin{array}{l}\text { MM 'Students' OR MM 'Business Students' OR MM ‘Classmates' OR MM 'College Students' OR MM ‘Dental } \\
\text { Students' OR MM 'Elementary School Students' OR MM 'Graduate Students' OR MM 'High School } \\
\text { Students' OR MM 'International Students' OR MM 'Junior High School Students' OR MM 'Kindergarten } \\
\text { Students' OR MM 'Law Students' OR MM 'Medical Students' OR MM 'Postgraduate Students' OR MM } \\
\text { 'Preschool Students' OR MM 'Reentry Students' OR MM 'Seminarians' OR MM 'Special Education Students' } \\
\text { OR MM 'Transfer Students' OR MM 'Vocational School Students' }\end{array}$ & 100,965 \\
\hline S9 & Student & 491,276 \\
\hline S8 & Youth & 67,771 \\
\hline S7 & Adolescent & 181,497 \\
\hline S6 & S1 OR S2 OR S3 OR S4 OR S5 & 45,897 \\
\hline S5 & 'cluster analysis' & 7344 \\
\hline S4 & 'factor analysis' & 36,938 \\
\hline S3 & 'latent class analysis' & 1106 \\
\hline S2 & MM 'Factor Analysis' OR MM 'Item Analysis (Statistical)' OR MM 'Statistical Rotation' & 9344 \\
\hline S1 & MM ‘Cluster Analysis' & 1894 \\
\hline
\end{tabular}


Appendix 2. Search terms from PsychInfo [Qualitative].

\begin{tabular}{|c|c|c|}
\hline & Search term & $\begin{array}{l}\text { Number of } \\
\text { articles retrieved }\end{array}$ \\
\hline S10 & S4 AND S5 AND S9 & 809 \\
\hline S9 & S6 OR S7 OR S8 & 399,366 \\
\hline S8 & youth & 68,413 \\
\hline S7 & adolescen* & 208,259 \\
\hline S6 & $\begin{array}{l}\text { DE 'Students' OR DE 'Business Students' OR DE 'Classmates' OR DE 'College Students' OR DE ‘Dental Students' } \\
\text { OR DE ‘Elementary School Students' OR DE 'Graduate Students' OR DE 'High School Students' OR DE } \\
\text { 'International Students' OR DE 'Junior High School Students' OR DE 'Kindergarten Students' OR DE 'Law } \\
\text { Students' OR DE 'Medical Students' OR DE 'Postgraduate Students' OR DE 'Preschool Students' OR DE } \\
\text { 'Reentry Students' OR DE 'Seminarians' OR DE 'Special Education Students' OR DE 'Transfer Students' OR } \\
\text { DE 'Vocational School Students' }\end{array}$ & 168,625 \\
\hline S5 & $\begin{array}{l}\text { DE 'Alcohol Drinking Attitudes' OR DE 'Alcohol Drinking Patterns' OR DE 'Alcohol Abuse' OR DE 'Alcohol } \\
\text { Intoxication' OR DE 'Social Drinking' }\end{array}$ & 30,844 \\
\hline S4 & S1 OR S2 OR S3 & 382,805 \\
\hline S3 & experiences & 371,601 \\
\hline S2 & $\begin{array}{l}\text { DE 'Interviews' OR DE 'Intake Interview' OR DE 'Interview Schedules' OR DE 'Job Applicant Interviews' OR DE } \\
\text { 'Psychodiagnostic Interview' }\end{array}$ & 9841 \\
\hline S1 & DE 'Qualitative Research' & 3916 \\
\hline
\end{tabular}

Appendix 3. Search terms from MEDLINE [Qualitative].

\begin{tabular}{lll}
\hline & Search term & \multicolumn{1}{c}{$\begin{array}{l}\text { Number of } \\
\text { articles retrieved }\end{array}$} \\
\hline S10 & S4 AND S5 AND S9 & 434 \\
S9 & S6 OR S7 OR S8 & $1,572,139$ \\
S8 & youth & 40,199 \\
S7 & MH 'Adolescent' & $1,542,922$ \\
S6 & MH 'Students' & 32,110 \\
S5 & MH 'Alcohol Drinking' & 48,505 \\
S4 & S1 OR S2 OR S3 & 138,689 \\
S3 & experiences & 371,601 \\
S2 & MH 'Qualitative Research' & 9841 \\
S1 & MH 'Focus Groups' & 3916 \\
\hline
\end{tabular}

Appendix 4. Search terms from CINAHL [Qualitative].

\begin{tabular}{lll}
\hline & Search term & $\begin{array}{l}\text { Number of } \\
\text { articles retrieved }\end{array}$ \\
\hline S10 & S4 AND S5 AND S9 & 566 \\
S9 & S6 OR S7 OR S8 & 205,214 \\
S8 & Youth & 12,723 \\
S7 & MH 'Adolescence+' & 193,078 \\
S6 & (MH 'Students, College') OR & 15,801 \\
& (MH 'Students, High School') & \\
S5 & MH 'Alcohol Drinking' & 11,518 \\
S4 & S1 OR S2 OR S3 & 142,547 \\
S3 & MH 'Audiorecording' & 26,162 \\
S2 & MH 'Qualitative Studies+' & 113,690 \\
S1 & MH 'Interviews+' & 64,969 \\
\hline
\end{tabular}

